\title{
Corticotrophin-releasing factor receptors within the ventromedial hypothalamus regulate hypoglycemia- induced hormonal counterregulation
}

\author{
Rory J. McCrimmon, ${ }^{1}$ Zhentao Song, ${ }^{2}$ Haiying Cheng,, Ewan C. McNay, ${ }^{1}$ \\ Catherine Weikart-Yeckel, ${ }^{1}$ Xiaoning Fan, ${ }^{1}$ Vanessa H. Routh, ${ }^{2}$ and Robert S. Sherwin ${ }^{1}$ \\ ${ }^{1}$ Department of Internal Medicine, Yale University School of Medicine, New Haven, Connecticut, USA. \\ 2Department of Pharmacology and Department of Physiology and Neurosciences, New Jersey Medical School, Newark, New Jersey, USA.
}

\begin{abstract}
Recurrent episodes of hypoglycemia impair sympathoadrenal counterregulatory responses (CRRs) to a subsequent episode of hypoglycemia. For individuals with type 1 diabetes, this markedly increases (by 25 -fold) the risk of severe hypoglycemia and is a major limitation to optimal insulin therapy. The mechanisms through which this maladaptive response occurs remain unknown. The corticotrophin-releasing factor (CRF) family of neuropeptides and their receptors (CRFR1 and CRFR2) play a critical role in regulating the neuroendocrine stress response. Here we show in the Sprague-Dawley rat that direct in vivo application to the ventromedial hypothalamus (VMH), a key glucose-sensing region, of urocortin I (UCN I), an endogenous CRFR2 agonist, suppressed $(-55-60 \%)$, whereas CRF, a predominantly CRFR1 agonist, amplified $(-50-70 \%)$ CRR to hypoglycemia. UCN I was shown to directly alter the glucose sensitivity of VMH glucose-sensing neurons in wholecell current clamp recordings in brain slices. Interestingly, the suppressive effect of UCN I-mediated CRFR2 activation persisted for at least 24 hours after in vivo $\mathrm{VMH}$ microinjection. Our data suggest that regulation of the CRR is largely determined by the interaction between CRFR2-mediated suppression and CRFR1-mediated activation in the VMH.
\end{abstract}

\section{Introduction}

Insulin therapy in type 1 diabetes carries with it the risk of iatrogenic severe hypoglycemia. This risk is further increased when glucose counterregulatory defense mechanisms are defective (1-3). Sympathoadrenal activation in response to hypoglycemia increases endogenous glucose production, decreases peripheral glucose utilization, and generates the symptoms that provide the warning of incipient hypoglycemia (2). In type 1 diabetes, this is the principal glucose CRR to progressing hypoglycemia. Single or recurrent episodes of hypoglycemia in human subjects have been shown to impair sympathoadrenal responses to a subsequent episode of hypoglycemia (4-6), a phenomenon that has also been demonstrated in rat models (7-9). Hypoglycemia, in effect, renders an individual increasingly susceptible to hypoglycemia, and this has become a major limitation to effective insulin therapy in type 1 diabetes.

To understand the mechanism through which hypoglycemia induces the state of defective glucose CRR, a greater understanding of the factors that modulate the CRR in key glucose-sensing regions is required. The detection of incipient hypoglycemia is thought to occur in specialized glucose-sensing neurons in the brain $(10-16)$ and periphery $(17,18)$. The brain glucose-sensing

Nonstandard abbreviations used: ACSF, artificial cerebrospinal fluid; aECF, artificial extracellular fluid; APF, action potential frequency; aSVG, anti-sauvagine; $\mathrm{CRF}$, corticotrophin-releasing factor; CRFR, CRF receptor; CRR, counterregulatory response; GE, glucose-excited; GI, glucose-inhibited; HPA, hypothalamic-pituitaryadrenal; IR, input resistance; MP, membrane potential; PVN, paraventricular nucleus; $\mathrm{UCN}$, urocortin; $\mathrm{VMH}$, ventromedial hypothalamus.

Conflict of interest: The authors have declared that no conflict of interest exists. Citation for this article: J. Clin. Invest. 116:1723-1730 (2006). doi:10.1172/JCI27775 region that has been most studied is the ventromedial hypothalamus (VMH). The VMH contains neurons that react to changes in circulating glucose (19-21) and has been shown to play a crucial role in the detection of incipient hypoglycemia and in the generation of a CRR $(8,10-12,22,23)$.

We have previously shown that corticotrophin-releasing factor (CRF), when delivered systemically, can induce the state of defective CRR to subsequent insulin-induced hypoglycemia (9). The CRF neuropeptide family (CRF and urocortins I, II, and III [UCN I-III]; ref. 24) are critical regulators of both neural and endocrine components of the stress response. In the brain, receptors for CRF, CRFR1 and CRFR2, show a distinct localization (24). CRF has a higher affinity for CRFR1 than for CRFR2. UCN I shows high affinity for both CRFR1 and CRFR2, whereas as UCN II and UCN III are selective for CRFR2 (24-26). It has been suggested that CRF, acting through CRFR1, has a stimulatory effect, whereas the UCNs, acting through CRFR2, have a suppressive effect on the stress response (24). The VMH contains both CRFR1 and, specifically, an abundance of CRFR2 (27). Given the critical role of the CRF family in regulating the neuroendocrine stress response, we predicted that it would also play a role in the regulation of the CRR to acute hypoglycemia. Here we report that CRFR2 receptor ligands have a marked and persisting suppressive effect, whereas the predominant CRFR1 receptor ligand, $\mathrm{CRF}$, has a stimulatory effect on the CRR to acute hypoglycemia.

\section{Results}

Role of CRFR1 and CRFR2 in regulating CRRs to bypoglycemia. Because CRF has a higher affinity for CRFR1 and UCN I has a 10- to 40fold greater affinity for CRFR2 $(24,25)$, we initially used these 


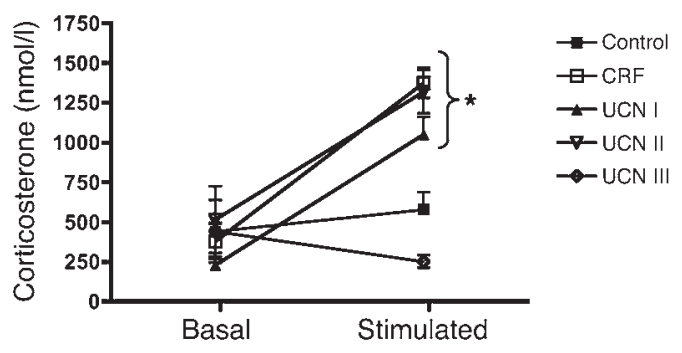

Figure 1

VMH CRF and UCN I-II, but not UCN III, stimulate corticosterone release under basal conditions. Microinjection of 4 pmol CRF (open squares), UCN I (filled triangles), and UCN II (inverted triangles), but not UCN III (diamonds) or control (filled squares) produced significant rises in plasma corticosterone levels. Values are shown as mean \pm SEM. ${ }^{\star} P<0.05$ versus control.

agents to examine the role of CRF receptors in the VMH in regulating the CRR to hypoglycemia. In the 60-minute period following microinjection into the $\mathrm{VMH}$, and prior to the start of the hyperinsulinemic hypoglycemic clamp procedure, both UCN I (4 pmol) and CRF ( $4 \mathrm{pmol})$ produced significant increases in circulating corticosterone levels (both, $P<0.05$; Figure 1 and Table 1 ) but had no significant effect on basal levels of epinephrine and glucagon (Table 1). This effect was not reversed by the selective CRFR2 antagonist anti-sauvagine (aSVG; Table 1).

Corticosterone levels remained high during the subsequent hypoglycemic clamp study; however, whereas UCN I resulted in a marked suppression of CRR to acute hypoglycemia (epinephrine: $\sim 55 \%$ reduction, $P<0.05$ versus control; and glucagon: $\sim 60 \%$ reduction, $P<0.05$ versus control; Figure $2, \mathrm{~A}-\mathrm{C}$ ), CRF ( 4 pmol) amplified epinephrine ( $~ 70 \%$ increase; $P<0.05$ versus control) and glucagon ( $\sim 55 \%$ increase; $P<0.05$ versus control) CRR to hypoglycemia (Figure 2, B and C). The suppressive effect of UCN I on CRR was reversed by combining UCN I with the selective CRFR2 antagonist aSVG (Figure 2; reversal of the UCN I effect with $20 \mathrm{pmol}$ aSVG $[P=$ NS versus control $]$ but not 4 pmol aSVG $[P<0.05$, versus control]). Interestingly, VMH microinjection of aSVG alone lead to a significant amplification of CRR $(P<0.05$ versus control; Figure 2, A-C), which is consistent with an endogenous ligand of CRFR2 in the VMH regulating sympathoadrenal responses during acute hypoglycemia. In further studies, we demonstrated that a 10 -fold increase $(40 \mathrm{pmol})$ in the dose of CRF resulted in the suppression of CRR to hypoglycemia [epinephrine, AUC/t $4.6 \pm 0.6$ (mean $\pm \mathrm{SEM}$ ) versus $2.5 \pm 0.5 \mathrm{nmol} / \mathrm{l}$; and glucagon, AUC/t $103.4 \pm 12.1$ versus $58.9 \pm 7.7 \mathrm{ng} / \mathrm{l}$; both $P<0.05$, control versus 40 pmol CRF]. This effect could be reversed with aSVG (20 pmol; data not shown), an effect that is consistent with results of in vitro studies showing that CRF at higher doses binds to CRFR2 (24). In contrast, plasma corticosterone rose to a similar level under all conditions (all $P=$ NS versus control; Figure 2D).

UCN II and III are 2 recently discovered additional members of the CRF family of neuropeptides (24). The CRF ligands differ in their affinity for CRFR1 and CRFR2 $(25,26)$. UCN I-III all show a higher affinity for CRFR2 than does CRF, with UCN II showing the highest binding affinity and UCN III being the most selective (25, $26)$. We found that both UCN II ( 70\% suppression of epinephrine response; $P<0.05$ versus control; data not shown) and UCN III (Figure 3, A-D) had a similar marked suppressive effect on CRR (both $P<0.05$ versus control). UCN II, but not UCN III, also evoked a corticosterone rise during the basal preclamp period $(P<0.05$ UCN II versus control; Figure 1).

CRFR2 ligands have a persisting suppressive effect on CRR to bypoglycemia. To explore the effect of prior activation of CRFR2 in the VMH on subsequent glucose CRR to hypoglycemia, nonfasted, chronically catheterized, freely mobile male Sprague-Dawley rats were microinjected with CRF (4 pmol), UCN I (4 pmol), or control solution 24 hours prior to performance of a hyperinsulinemic hypoglycemic clamp study. Injection of rats with UCN I, in comparison with both CRF and the control, had a marked suppressive effect on CRR to subsequent hypoglycemia $(P<0.05$ for epinephrine, glucagon, and glucose infusion rate [GIR] during hypoglycemia versus control; Figure 4, A-C). The requirement for exogenous glucose was increased, and epinephrine and glucagon secretory responses were blunted. Basal corticosterone levels 24 hours following microinjection did not differ between groups and rose to a similar degree in all groups during subsequent hypoglycemia (Figure 4D).

UCN alters thresholds for activation of glucose-sensing neurons. Two types of glucose-sensing neurons in the VMH are directly regulated by changes in extracellular glucose. Glucose-excited (GE) neurons increase and glucose-inhibited (GI) neurons decrease their action potential frequency (APF) as ambient glucose levels rise $(21,28,29)$. To determine whether UCN I directly altered the glucose sensitivity of VMH glucose-sensing neurons, we next performed whole-cell current clamp recordings in VMH GI and GE neurons in brain slices (Figures 5 and 6). We have previously shown that control VMH GI and GE neurons are extremely sensitive to decreases in extracellular glucose levels below $1 \mathrm{mM}$ (20). Figure 5A illustrates a control VMH GI neuron in which APF, membrane potential (MP), and input resistance (IR) were reversibly increased as glucose levels decreased to $0.5 \mathrm{mM}$ (top trace) or $0.1 \mathrm{mM}$ (bottom trace). In contrast, after 15 minutes incubation of brain slices with $0.2 \mu \mathrm{M}$ UCN I, MP, APF and IR reversibly increased only when glucose levels decreased from 2.5 to $0.1 \mathrm{mM}$ (Figure 5B, upper trace) but not from 2.5 to $0.5 \mathrm{mM}$ (Figure 5B, lower trace). The percent increase in IR as extracellular glucose levels were decreased from 2.5 to $0.5 \mathrm{mM}$ was significantly greater in control $(28.9 \% \pm 5.2 \% ; n=11)$ than in UCN I-pretreated $(-1.7 \% \pm 2.7 \% ; n=13)$ VMH GI neurons $(P<0.0001$; Figure $5 D)$. Figure $5 \mathrm{C}$ shows that when VMH GI neurons were pretreated with both UCN I and aSVG, APF, MP, and IR were reversibly increased when glucose levels were decreased from 2.5 to $1,0.5$, or $0.1 \mathrm{mM}$.

\section{Table 1}

Effect of VMH microinjection of each solution under basal conditions on epinephrine, glucagon, and corticosterone levels

$\begin{array}{lccc}\begin{array}{l}\text { Group } \\ \text { (agent-dose in pmol) }\end{array} & \begin{array}{c}\text { Epinephrine } \\ \text { (nmol/l) }\end{array} & \begin{array}{c}\text { Glucagon } \\ \text { (ng/l) }\end{array} & \begin{array}{c}\text { Corticosterone } \\ \text { (nmol/l) }\end{array} \\ \text { aECF }(n=12) & -0.04(0.08) & 1.2(2.7) & 205(84) \\ \text { UCN I-4 }(n=6) & -0.15(0.16) & -9.3(3.0) & 861(133)^{\mathrm{A}} \\ \text { UCN I-4 + aSVG-4 }(n=6) & 0.08(0.15) & 9.5(6.9) & 835(173)^{\mathrm{A}} \\ \text { UCN I-4 + aSVG-20 }(n=6) & 0.14(0.07) & 2.5(2.9) & 913(176)^{\mathrm{A}} \\ \text { CRF-4 }(n=7) & 0.29(0.30) & 12.2(5.9) & 1,156(127)^{\mathrm{A}} \\ \text { CRF-40 }(n=6) & 0.20(0.05) & 5.2(4.0) & 1,098(104)^{\mathrm{A}} \\ \text { CRF-40 + aSVG-20 }(n=3) & 0.13(0.03) & 3.7(1.5) & 1,407(384)^{\mathrm{A}} \\ \text { aSVG-20 }(n=6) & 0.12(0.07) & 7.6(5.4) & 549(171) \\ \end{array}$

Values are shown as mean (SEM) of change from baseline. ${ }^{A} P<0.05$ versus aECF (control). 

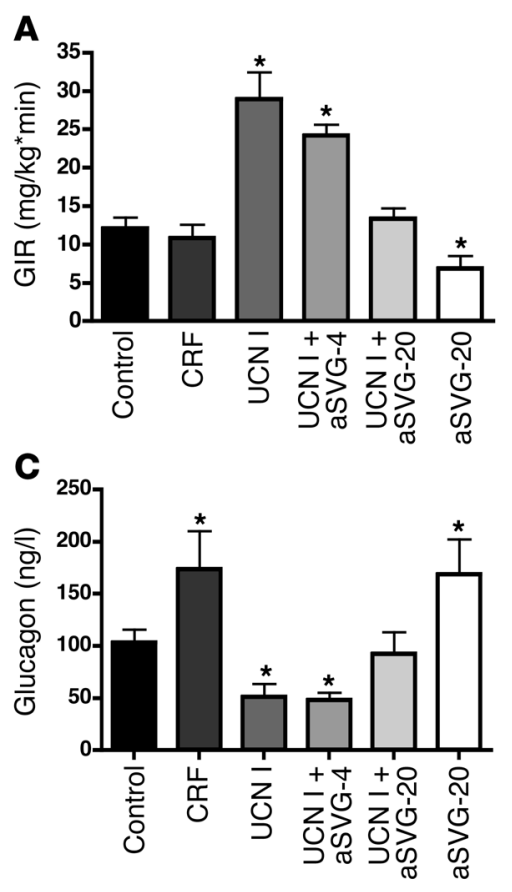

B

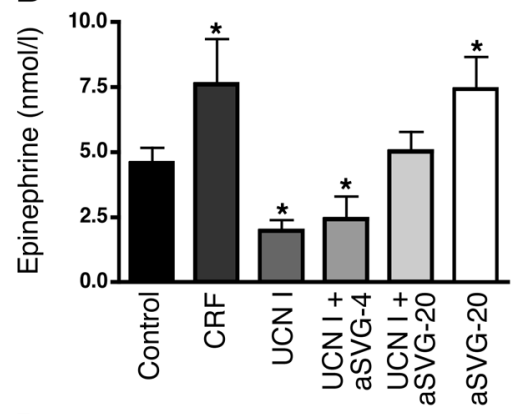

D

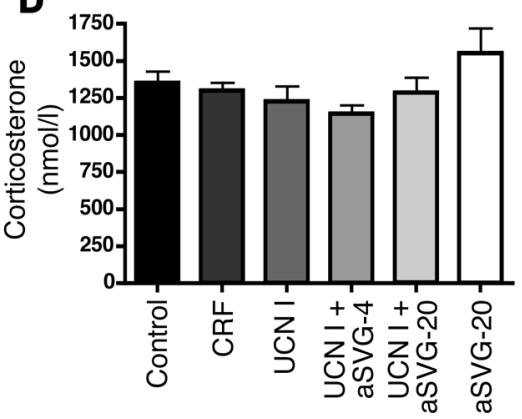

Figure 2

$\mathrm{VMH}$ microinjection of UCN I suppresses glucose CRRs to acute hypoglycemia, an effect that is reversed by the CRFR2 antagonist aSVG. (A-C) Four picomoles of UCN I suppressed the epinephrine and glucagon responses to acute hypoglycemia and increased requirements for exogenous glucose, an effect that was reversed by 20 pmol aSVG (UCN I + aSVG-20) but not 4 pmol aSVG (UCN I + aSVG-4). aSVG alone produced an amplification of glucose CRRs. In contrast, an equivalent dose of CRF amplified hormonal counterregulatory responses to acute hypoglycemia. (D) No effect was seen on subsequent corticosterone responses to hypoglycemia. Values shown as mean \pm SEM. Hormone values represent $\mathrm{AUC} / \mathrm{t}$ and glucose infusion rate (GIR) mean rates over $60-120$ minutes. ${ }^{*} P<0.05$ versus control.
Finally, an increase in IR (due to $\mathrm{Cl}^{-}$channel closure) as the glucose level decreases is a reliable index of the degree of activation of VMH GI neurons. The percent change in IR in response to decreasing the glucose level from 2.5 to $0.5 \mathrm{mM}$ was significantly greater for the UCN plus aSVG-pretreated GI neurons than for those pretreated with UCN alone $(14.1 \% \pm 5.5 \% ; n=3 ; P=0.02$; Figure 5D). There was no significant difference in the percent change in IR between control and UCN I plus aSVG-pretreated VMH GI neurons (Figure 5D). Although GE neurons were observed less frequently than GI neurons, we noted a similar decrease in glucose sensitivity. Figure 6A shows a control GE neuron in which APF, MP, and IR were reversibly decreased when glucose levels were lowered to either 0.1 or $0.5 \mathrm{mM}$. In contrast, when the brain slice was incubated in $0.2 \mu \mathrm{M}$ UCN I, APF, MP, and IR were only decreased when glucose levels were lowered to 0.1 but not $0.5 \mathrm{mM}(n=2$; Figure $6 \mathrm{~B})$. As with the GI neurons, this effect was reversed by pretreatment of brain slices with UCN I plus $1 \mu \mathrm{M}$ aSVG (Figure 6C).

\section{Discussion}

The VMH is probably the best characterized of the central glucose-sensing regions involved in hypoglycemia detection and clearly contains neurons that react to changes in circulating glucose levels (19-21). The mechanisms used by glucose-sensing neurons to detect fluctuations in systemic glucose levels remain to be fully elucidated, although they appear to show considerable similarity to that of the pancreatic $\beta$ cell in that glucokinase $(15,30)$ and the ATP-sensitive potassium channel $(8,22)$ are key links through which the change in systemic glucose is translated into a change in neuronal firing rate. While other brain regions such as the hindbrain (31) and peripheral glucose sensors $(17,18)$ undoubtedly play a role in hypoglycemia detection and may in fact form an integrated glucose-sensing system, the VMH probably plays the major role. Infusing glucose directly into the $\mathrm{VMH}$ through reverse microdialysis during systemic hypoglycemia nearly abolishes CRRs (12). In this context, any mechanism regu-

\section{Figure 3}

VMH UCN III suppresses glucose CRRs to acute hypoglycemia. Four picomoles of UCN III (black bars) suppressed the epinephrine and glucagon responses to acute hypoglycemia and increased requirements for exogenous glucose (A-C) but had no effect on corticosterone responses (D). Values are shown as mean \pm SEM. Hormone values represent $A U C / t$ and GIR mean rates over $60-120$ minutes. ${ }^{*} P<0.05$ versus control.
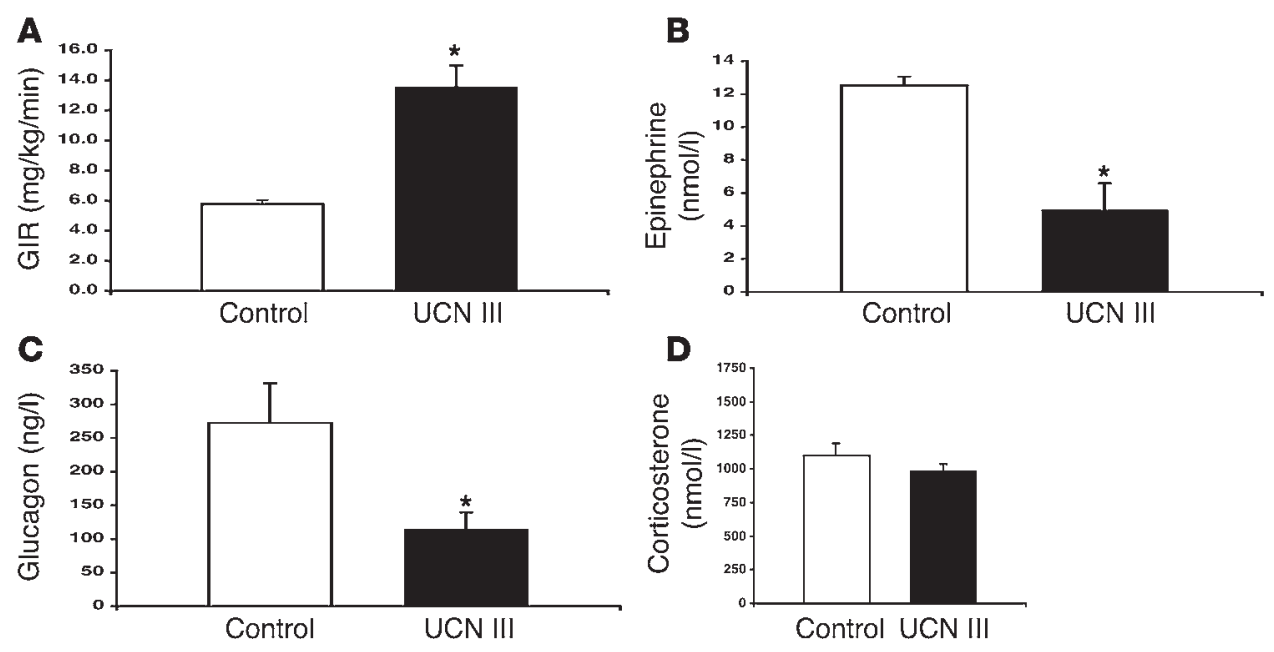

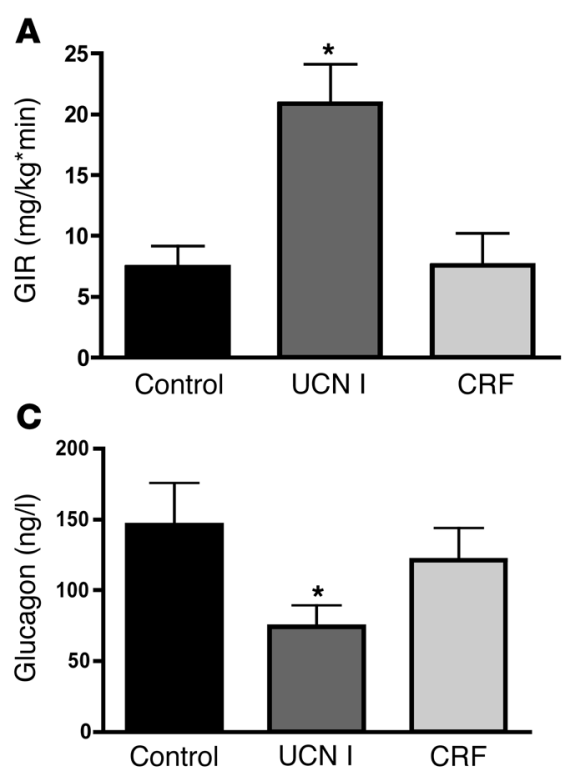
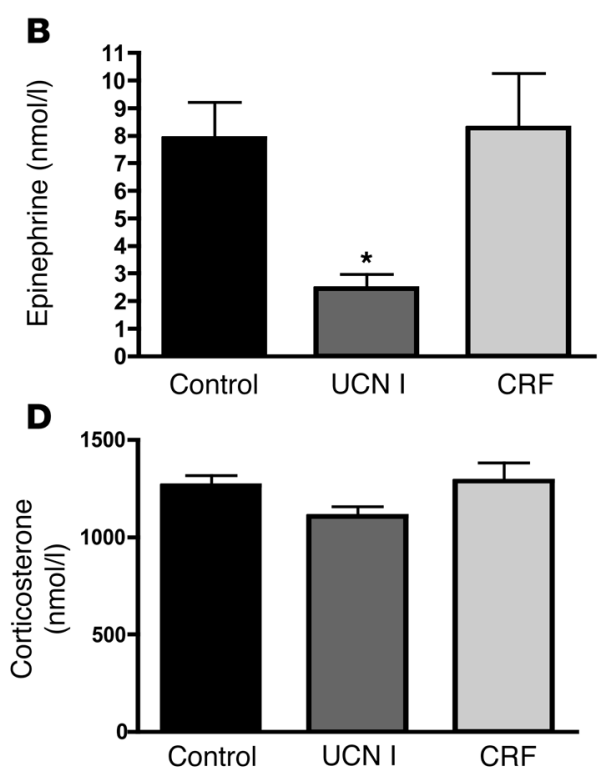

Figure 4

The suppressive effect of $\mathrm{VMH}$ UCN I on CRRs to hypoglycemia persists for at least 24 hours. (A-C) UCN I, but not CRF, suppressed the epinephrine and glucagon responses to acute hypoglycemia and increased requirements for exogenous glucose. (D) No effect was seen on subsequent corticosterone responses to hypoglycemia. Values are shown as mean \pm SEM. Hormone values represent AUC/t and GIR mean rates over 60-120 minutes. ${ }^{*} P<0.05$ versus control. lating sympathoadrenal responses to hypoglycemic stress within the VMH is likely to have major effects on the overall CRR.

Our data suggest that such a system, based on the interaction between CRFR1- and CRFR2-mediated effects, exists within the $\mathrm{VMH}$, and, consistent with the major role that the VMH plays in hypoglycemia sensing, we have shown that manipulation of this system has a marked effect on CRR to hypoglycemia. It has been recognized for many years that CRF plays a major role in regulating the neuroendocrine response to acute stress (24). The actions of CRF are mediated by its 2 receptor subtypes, CRFR1 and CRFR2 (24). UCN I-III are recently identified additional members of the CRF family of peptides. UCN I binds with high affinity to both CRFR1 and CRFR2 (24), whereas UCN II and UCN III are more selective for CRFR2 $(24,26,32,33)$. Interestingly, the UCNs all show a distinct distribution pattern in the brain, and they tend to colocalize with CRFR2 $(24,26,32,33)$. It has been proposed that the UCNs are the endogenous ligands for CRFR2 and may serve to modulate the stimulatory effects of the CRF-CRFR1 coupling on the stress response $(24,34)$. Certainly, CRFR2-KO mice exhibit greater anxiety and show an exaggerated response to stress, whereas CRFR1-KO mice exhibit less anxiety and have reduced responses to stress $(34,35)$.

The unique distribution pattern of UCN I-III and CRFR2 also suggest that each peptide has its own physiological significance. Few groups have, however, manipulated CRFRs within discrete brain regions, although it was recently reported that UCN II inhibited dorsal raphe 5-hydroxytryptamine neurons, an effect that was reversed by aSVG (36). Both CRFR1 and CRFR2 are expressed within the VMH, with the relative abundance of CRFR2 being much greater (27). Recently, it has also been shown that UCN III nerve terminals are present in the VMH (32). In our study, microinjection of CRFR2 activators (UCN I-III and high-dose CRF) into the $\mathrm{VMH}$ had a marked suppressive effect on the CRR to acute hypoglycemia, an effect that was reversed when they were combined with the selective CRFR2 antagonist aSVG. In contrast, microinjection of an equimolar dose of CRF (which would be expected to act predominantly through CRFR1; ref. 24) amplified CRRs. Moreover, microinjection of aSVG alone also led to an amplification of the CRR to hypoglycemia, a finding that suggests both the presence of an endogenous ligand and that reduced CRFR2 tone contributes to an amplified stress response. At this point, the lack of a specific CRFR1 antagonist that is suitable for direct microinjection to specific brain nuclei in vivo limits our examination of the counteracting effects CRFR1 and CRFR2 within the VMH. However, by examining the effect of each member of the CRF family of neuropeptides, and using their differing affinities for CRFR1 and CRFR2, our results as a whole support the concept that the balance between CRFR2-mediated inhibition and CRFR1mediated activation in the VMH determine, to a large extent, the magnitude of the CRR to acute hypoglycemia.

The effect of UCN I-III and CRF to evoke a rise in plasma corticosterone levels following VMH microinjection was unanticipated. Because all of the CRF peptides except UCN III stimulated corticosterone release, and the effect was not reversed by aSVG, our data strongly suggest this is a CRFR1-mediated effect. It is well established the CRF released by the paraventricular nucleus (PVN) activates CRFR1 receptors in the anterior pituitary, resulting in adrenocorticotrophin hormone (ACTH) release. ACTH in turn stimulates corticosterone release from the adrenal cortex. CRFR1 in the hypothalamus is found mainly in the dorsomedial hypothalamus (DMH), to a lesser extent in the $\mathrm{VMH}$ and supraoptic nucleus (SON), and to an even smaller degree in the PVN (27). In contrast, CRFR2 expression is mainly localized to the VMH, with much less expression seen in the SON and PVN (27). Anatomically, these regions are to a varying degree interconnected with the principal pathway from $\mathrm{VMH}$ to PVN thought to run indirectly via the $\mathrm{DMH}$ (37). The receptor distribution, taken together with the findings from the present study, provide support for a CRFR1mediated stimulatory pathway leading from the VMH to the PVN, ultimately evoking corticosterone release.

Glucose-sensing neurons react to changes in the intraneuronal energy status (28). Some groups have proposed that recurrent hypoglycemia induces changes that increase the delivery of glucose $(38,39)$ and/or alternate fuels $(40,41)$ to those neurons and thus impairs their ability to recognize a subsequent fall in blood glucose. This in turn leads to reduced or defective CRR to hypoglycemia. However, the presence of a mechanism within the 
A

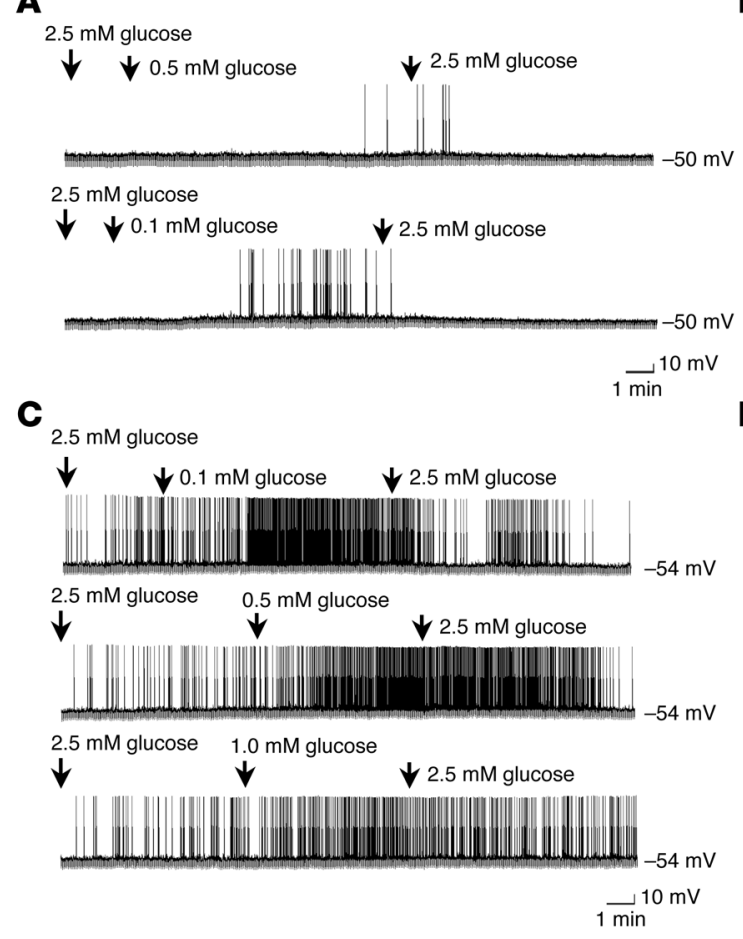

B $2.5 \mathrm{mM}$ glucose

$\downarrow \downarrow 0.1 \mathrm{mM}$ glucose $\quad \downarrow^{2.5 \mathrm{mM} \text { glucose }}$

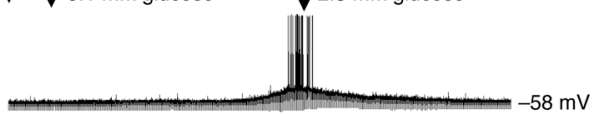

$2.5 \mathrm{mM}$ glucose

$\downarrow \downarrow 0.5 \mathrm{mM}$ glucose $\quad \downarrow 2.5 \mathrm{mM}$ glucose
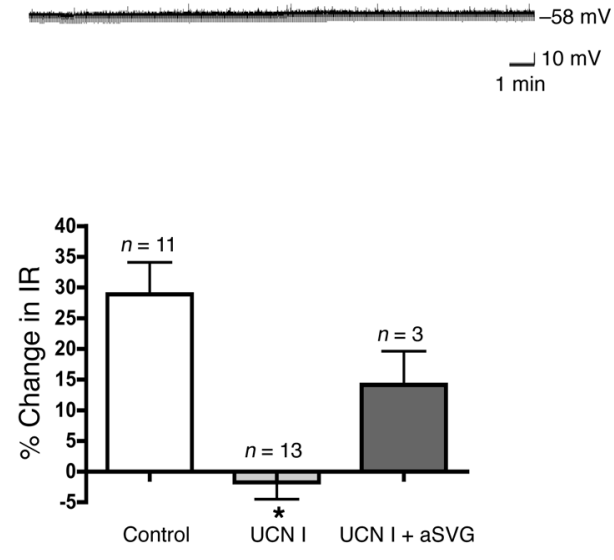

\section{Figure 5}

Consecutive whole-cell current clamp recordings from VMH GI neurons. Resting membrane potential (RMP) is noted to the right of each trace. The downward deflections represent the membrane voltage response to a constant hyperpolarizing pulse. (A) Under control conditions, RMP, $\mathrm{APF}$, and IR reversibly increased when glucose levels decreased from 2.5 to 0.5 (upper trace) or $0.1 \mathrm{mM}$ (lower trace). (B) Following pretreatment of the brain slice with $0.2 \mu \mathrm{M} \cup C N \mathrm{I}, \mathrm{MP}, \mathrm{APF}$, and IR reversibly increased only when glucose levels decreased from 2.5 to $0.1 \mathrm{mM}$ (upper trace) but not from 2.5 to $0.5 \mathrm{mM}$ (lower trace; $n=9$ ). (C) Pretreatment of the brain slices with $0.2 \mu \mathrm{M}$ UCN I plus $1 \mu \mathrm{M}$ aSVG reversibly increased MP, APF, and IR when glucose levels decreased from 2.5 to 0.1 (upper trace), 0.5 (middle trace), and $1 \mathrm{mM}$ (lower trace). (D) UCN I significantly reduced the increased IR in response to decreased extracellular glucose from 2.5 to $0.5 \mathrm{mM}$ (control, $28.9 \% \pm 5.2 \%$ versus $\mathrm{UCN},-1.7 \% \pm 2.7 \%$; $P<0.0001$ ). aSVG reversed this effect (UCN plus aSVG, $14.1 \% \pm 5.5 \%$ versus UCN, $-1.7 \% \pm 2.7 \% ; P=0.02$ ).

VMH to regulate the magnitude of the stress response also implies that a defect within this system might contribute to the defect in hormonal counterregulation that arises subsequent to recurrent hypoglycemia (i.e., the ability of glucose-sensing neurons to alter their firing rate in response to the hypoglycemic signal may be impaired). While the ability to generate a stress response is essential to an organism's ability to adapt, the stress response itself may have negative consequences for the organism. The effect of chronic stress in downregulating the hypothalamic-pituitary-adrenal (HPA) axis is well established and has been studied extensively. In contrast, much less is known about the effect of chronic or recurrent stress on the sympathoadrenal stress response. In this context, recurrent hypoglycemia provides an interesting physiological model in that the predominant defect to arise from recurrent hypoglycemia appears to be a defective sympathoadrenal, rather than HPA, response to stress.

The mechanism through which this downregulation occurs is unknown. In a series of elegant studies, Davis and colleagues have suggested that hypoglycemia-induced rises in cortisol $(42,43)$ acting centrally (44) might be responsible for the downregulation of the sympathoadrenal stress response to hypoglycemia; however, others $(9,45,46)$ have been unable to demonstrate similar effects. We reported in an earlier study in a rat model that recurrent systemic delivery of CRF in a dose designed to evoke corticosterone respons- es similar to that seen during acute hypoglycemia suppressed the sympathoadrenal response to subsequent hypoglycemia, whereas recurrent systemic corticosterone actually produced a trend toward an enhancement of the sympathoadrenal response (9). In contrast, in the present study, we found CRF delivered directly to the VMH actually amplified the CRR to acute hypoglycemia and when given 24 hours before had no significant effect on CRR responses. There were, however, marked differences in study design; Flanagan et al. (9) gave CRF systemically over 3 days, whereas we provided a single dose directly to the $\mathrm{VMH}$ on either the acute study day or 24 hours previously. CRF, if given as an acute intracerebroventricular (i.c.v.) injection, has been shown to increase plasma catecholamine and glucagon levels (47), but when it is given chronically i.c.v., neuroendocrine responses to $\mathrm{CRH}$ diminish over time (48). Moreover, the possibility of an effect of repeated CRH delivery to downregulate adrenal CRFR1 receptors cannot be excluded from our original study (24). In this context, our data would suggest that an alteration in the interplay between CRFR1-mediated activation and CRFR2-mediated suppression may contribute to the CRR defect that arises subsequent to recurrent hypoglycemia.

Our electrophysiological data provide further support for a role of $\mathrm{VMH}$ glucose-sensing neurons in hypoglycemia detection and activation of the HPA axis. We show here that CRFR2 activation blunts both the hormonal response to hypoglycemia and the glu- 
A

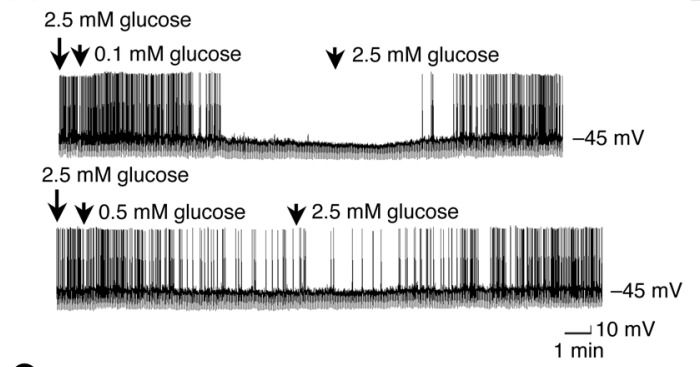

C

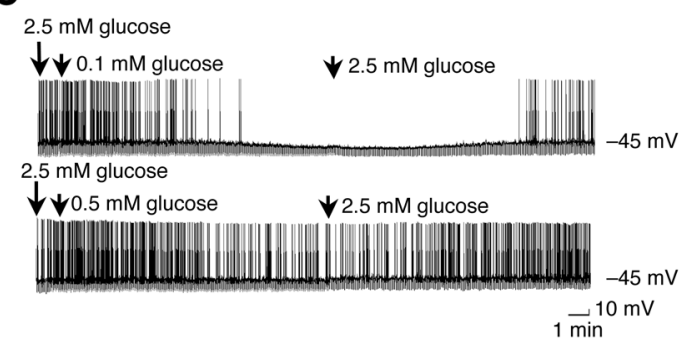

B

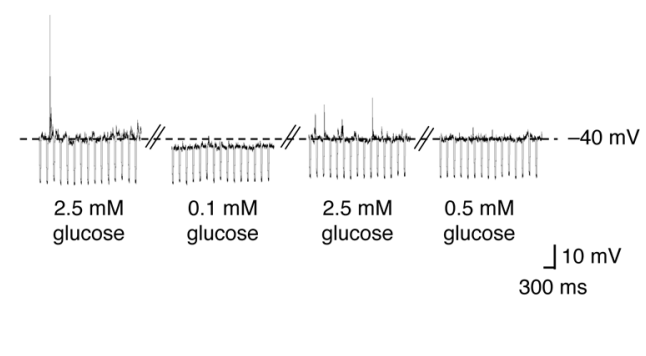

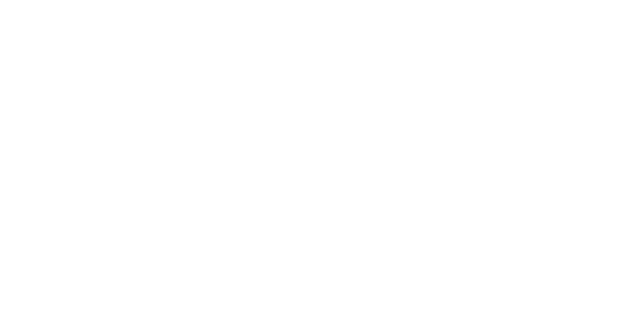

\section{Figure 6}

Effects of UCN I on VMN GE neurons. (A and C) Consecutive whole-cell current clamp recordings in VMN GE neurons. (B) Segments from consecutive whole-cell current-clamp recordings in a VMN GE neuron illustrated in an expanded time scale. RMP is noted to the right of each trace in A-C. The downward deflections represent the membrane voltage response to a constant hyperpolarizing pulse. (A) Under control conditions, RMP, APF, and IR reversibly decreased when glucose levels decreased from 2.5 to 0.1 (upper trace) or 0.5 mM (lower trace). (B) Following treatment of the brain slice with $0.2 \mu \mathrm{M} U \mathrm{CN}, \mathrm{MP}, \mathrm{APF}$, and IR reversibly decreased only when glucose levels decreased from 2.5 to $0.1 \mathrm{mM}$ but not from 2.5 to $0.5 \mathrm{mM}$. (C) When the brain slice was pretreated with $0.2 \mathrm{mM}$ UCN and $1 \mathrm{mM}$ aSVG, MP, APF, and IR reversibly decreased when glucose levels were decreased to 0.1 (upper trace) and $0.5 \mathrm{mM}$ (lower trace), as observed for control conditions.

cose sensitivity of VMH GE and GI neurons. It is very likely that CRFR2 activation directly decreases the glucose sensitivity of these neurons. This is because the UCN cell bodies are found in the perifornical area lateral to the PVN, and it is their nerve terminals that are found in the $\mathrm{VMH}(32)$. We feel it is very unlikely that a change in glucose sensitivity of the postsynaptic glucose-sensing neurons in the VMH could be presynaptically mediated by UCN, since the $\mathrm{PVN}$ is not in the VMH slices. Moreover, we have extensive experience with the effects of glucose on GE and GI neurons (20, 21, 49). It is clear that these effects are mediated by ion channels that respond to metabolites of glucose rather than neurotransmitters. Thus, it is very unlikely that $\mathrm{UCN}$ is altering glucose sensitivity via effects on neurons upstream from GE and GI neurons. One caveat is that UCN may activate a neuron in the slice preparation that in turn releases a transmitter that then binds to a $G$ protein-coupled receptor on the glucose-sensing neuron. Subsequent activation of a signaling cascade could potentially alter the glucose sensitivity of metabolically sensitive ion channels. However, in either case, the effect of UCN is most likely to reflect a more long-term intraneuronal change, given that it does not reverse on wash-out of UCN. Whether this is a direct effect on the glucose-sensing neuron or a result of CRFR2-mediated neurotransmitter release on an upstream neuron, acting through a $G$ protein-coupled receptor-induced change in gene expression, the ultimate effect is a direct alteration of the metabolic sensitivity of these specialized neurons. Finally, our data showing that both GE and GI neurons were affected by CRFR2 activation might seem counterintuitive. However, there are several possible explanations. For example, it is possible that GE and GI neurons control different aspects of HPA axis activation that may require excitation or disinhibition, respec- tively. It is also possible that they function in a balance much like CRFR1 and CRFR2. Last, it is possible that only 1 of these circuits (GE- or GI-mediated) is active during hypoglycemia. These questions are the subject for further studies.

Hypoglycemia remains the principal limitation to optimal glucose-lowering therapy and is a major clinical problem for individuals with type 1 diabetes and the physicians who provide their care. The risk of severe hypoglycemia in such individuals is greatly increased because of defective counterregulatory defense mechanisms. The findings from the present study provide novel insights into a mechanism for regulating the neural sympathoadrenal response to hypoglycemic stress within a key glucose-sensing region, the VMH. Our data suggest that the interplay between CRFR1- and CRFR2-mediated actions in the VMH to a large extent determine the magnitude of the CRR to acute hypoglycemia. We demonstrate that this effect may occur, at least in part, through an alteration in the thresholds at which VMH glucose-sensing neurons become activated. Moreover, we demonstrate a persisting effect of CRFR2 modulation in the VMH on CRR to hypoglycemia. Future studies designed to determine the impact of recurrent hypoglycemia on CRFR1/CRFR2 within the VMH may help us to understand why individuals with type 1 diabetes develop defective hormonal CRRs over time.

\section{Methods}

Animals. Male Sprague-Dawley rats (mean \pm SEM weight, $305 \pm 4 \mathrm{~g}$ ) were housed in the Yale Animal Resource Center, fed a standard pellet diet (Agway Prolab 3000), and maintained on a 12-hour light/12-hour dark cycle. The animal care and experimental protocols were reviewed and approved by the Yale University Institutional Animal Care and Use Committee. 
One week prior to each study, all animals were anesthetized with an i.p. injection $(1 \mathrm{ml} / \mathrm{kg})$ of a mixture of xylazine $(20 \mathrm{mg} / \mathrm{ml}$; AnaSed, Lloyd Laboratories Inc.) and ketamine (100 mg/ml; Ketaset, Wyeth) at a ratio of 1:2 ( $\mathrm{vol} / \mathrm{vol}$ ), prior to undergoing vascular surgery for the implantation of vascular catheters in a carotid artery and jugular vein. Following this, microinjection guide cannulas were bilaterally inserted into the $\mathrm{VMH}$ (coordinates from bregma: $\mathrm{AP}-2.6 \mathrm{~mm}, \mathrm{ML} \pm 3.8 \mathrm{~mm}$, and $\mathrm{DV}-8.3 \mathrm{~mm}$ at an angle of $20^{\circ}$ ), as described previously $(11,12)$.

Microinjection. Sixty minutes prior to starting the hyperinsulinemic glucose clamp, 26-gauge microinjection needles, designed to extend $1 \mathrm{~mm}$ beyond the tip of the guide cannula (Plastics One Inc.), were inserted through the guide cannula bilaterally into each ventromedial hypothalamus. The study rat was then microinjected, using a CMA-102 infusion pump (CMA Microdialysis), over 2 minutes at a rate of $0.1 \mu \mathrm{l} / \mathrm{min}$ with either: UCN I (4 pmol), UCN II (4 pmol), UCN III (4 pmol), aSVG (4 or 20 pmol), CRF (4 or 40 pmol), artificial extracellular fluid (aECF; control), or combinations of the above. aSVG is a highly selective and potent CRFR2 antagonist (25). All chemicals were purchased from Sigma-Aldrich and dissolved in aECF. Following microinjection, the needles were left in place for 3 minutes before being removed. At the end of the study, the rats were euthanized and probe position confirmed in all rats histologically. In a separate group of rats, UCN I (4 pmol), CRF ( 4 pmol), or aECF (control) was microinjected as described above but 24 hours prior to the hyperinsulinemic hypoglycemic study.

Infusion protocol. In all experiments the same infusion protocol was used. All animals were fasted overnight. On the morning of the study, the vascular catheters were opened and maintained patent by a slow infusion of saline $(20 \mu \mathrm{l} / \mathrm{min})$. During the first 90 minutes, animals were allowed to settle and recover from any stress of handling. Sixty minutes prior to the commencement of the hyperinsulinemic glucose clamp, each animal was microinjected as described above. Thereafter, a hyperinsulinemic hypoglycemic clamp, as adapted for the rat (7), was used to provide a standardized hypoglycemic stimulus. At time 0, a 120 -minute $20 \mathrm{mU} / \mathrm{kg} / \mathrm{min}$ infusion of human regular insulin (Eli Lilly \& Co.) was begun. The plasma glucose level was allowed to fall to approximately $2.8 \mathrm{mmol} / \mathrm{l}(50 \mathrm{mg} / \mathrm{dl})$ and was then maintained at this level for 120 minutes using a variable-rate $20 \%$ dextrose infusion based on frequent plasma glucose determinations. Samples for measurement of the hormones epinephrine, glucagon, and insulin were taken at -60, 0, 60, 90, and 120 minutes. Red cells, after removal of plasma, were resuspended in sterile artificial plasma and then reinfused to prevent volume depletion and anemia.

Preparation of brain slices. Male 14- to 21-day-old Sprague-Dawley rats were obtained from colonies at the Veterans Affairs Medical Center in East Orange, New Jersey, USA. Animals were housed with their dams on a 12-hour light/12-hour dark cycle at $22-23^{\circ} \mathrm{C}$ and fed a low-fat diet $(5001$ Rodent Diet; Purina Mills Inc.) and water ad libitum. On the day of experiment, rats were anesthetized with ketamine/xylazine (80:10 mg/kg, i.p.) and transcardially perfused with ice-cold oxygenated $\left(95 \% \mathrm{O}_{2} / 5 \% \mathrm{CO}_{2}\right)$ perfusion solution composed of the following (in $\mathrm{mM}$ ): $2.5 \mathrm{KCl}, 7 \mathrm{MgCl}_{2}$, $1.25 \mathrm{NaH}_{2} \mathrm{PO}_{4}, 28 \mathrm{NaHCO}_{2}, 0.5 \mathrm{CaCl}_{2}, 7$ glucose, 1 ascorbate, 3 pyruvate (osmolarity adjusted to $\sim 300 \mathrm{mOsm}$ with sucrose; $\mathrm{pH}$ 7.4). Brains were rapidly removed and placed in ice-cold (slushy) oxygenated perfusion solution. Sections $(350 \mu \mathrm{m})$ through the hypothalamus were made on a vibratome (Vibroslice; Campden Instruments Ltd.). The brain slices were maintained at $34^{\circ} \mathrm{C}$ in oxygenated artificial cerebrospinal fluid (ACSF [in
$\mathrm{mM}$ ]: $126 \mathrm{NaCl}, 1.9 \mathrm{KCl}, 1.2 \mathrm{KH}_{2} \mathrm{PO}_{4}, 26 \mathrm{NaHCO}_{3}, 10$ glucose, $2.4 \mathrm{CaCl}_{2}$, $1.3 \mathrm{MgCl}_{2}$; osmolarity adjusted to $\sim 300 \mathrm{mOsm}$ with sucrose; $\mathrm{pH} 7.4$ ] for 15 minutes and allowed to come to room temperature. Slices were transferred to normal oxygenated ACSF ( $2.5 \mathrm{mM}$ glucose) containing either $0.2 \mu \mathrm{M}$ UCN or UCN plus aSVG $(1 \mathrm{mM})$ for 15 minutes and then maintained in normal oxygenated ACSF for the remainder of the day.

Electrophysiology. Viable neurons were visualized and studied under infrared differential-interference contrast microscopy using a Leica Microsystems DM LFS microscope equipped with a $\times 40$ long-working-distance water-immersion objective as described previously (5). Current clamp recordings (standard whole-cell recording configuration) from neurons in the VMH were performed using a MultiClamp 700A (Axon Instruments) and analyzed using pCLAMP 9 software. During recording, brain slices were perfused at $10 \mathrm{ml} / \mathrm{min}$ with normal oxygenated ACSF. Borosilicate pipettes (1-3 M $\Omega$; Sutter Instrument) were filled with an intracellular solution containing (in $\mathrm{mM}$ ): 128 potassium gluconate, $10 \mathrm{KCl}, 4 \mathrm{KOH}, 10$ HEPES, $4 \mathrm{MgCl}_{2}, 0.5 \mathrm{CaCl}_{2}, 5$ EGTA, and $2 \mathrm{Na}_{2} \mathrm{ATP}$; pH 7.2. Osmolarity was adjusted to $290-300 \mathrm{mOsm}$ with sucrose. Junction potential between the bath and the patch pipette was nulled before the formation of a G $\Omega$ seal. Membrane potential and APF were allowed to stabilize for 10-15 minutes after the formation of the whole-cell configuration. Neurons whose access resistance exceeded $20 \mathrm{M} \Omega$ after this time were rejected. IR was calculated from the change in membrane potential measured, during the last 1 minute, of a small 500-ms hyperpolarizing pulse ( $-20 \mathrm{pA})$ given every 3 seconds. Extracellular glucose levels were altered as described in the figures.

Analytical procedures. Plasma levels of glucose were measured by the glucose oxidase method (Beckman Coulter). Catecholamine analysis was performed by HPLC using electrochemical detection (ESA); plasma insulin and glucagon levels were measured by RIA (Linco Research Inc.). All data are expressed as the mean \pm SEM. Area under the curve (AUC) for each hormone was calculated for each individual study and then divided by time of study (120 minutes). Means from each group were then compared using repeated-measures ANOVA or a 2-tailed Student's $t$ test as appropriate (SPSS 11.0 for Windows). $P$ values less than 0.05 were considered statistically significant.

\section{Acknowledgments}

This work was supported by a research grant from the NIH (DK-20495, DK-55619, and DK-64566) as well as the Juvenile Diabetes Research Foundation Center for the Study of Hypoglycemia at Yale University and the NIDDK-supported Diabetes Endocrinology Research Center. R.J. McCrimmon is the recipient of a Career Development Award from the Juvenile Diabetes Research Foundation. The authors are grateful to Xiaoning Fan, Yuyan Ding, Ralph Jacob, Aida Groszmann, and Andrea Belous for technical support and assistance.

Received for publication December 23, 2005, and accepted in revised form March 14, 2006.

Address correspondence to: Rory J. McCrimmon, Yale University School of Medicine, Department of Internal Medicine, PO Box 208020, New Haven, Connecticut 06520-8020, USA. Phone: (203) 785-4664; Fax: (203) 737-5558; E-mail: rory.mccrimmon@yale.edu.
1. Cryer, P.E. 1992. Iatrogenic hypoglycemia as a cause
of hypoglycemia-associated autonomic failure in
IDDM. A vicious cycle. Diabetes. 41:255-260.
2. Gerich,J.E., Mokan, M., Veneman, T., Korytkowski, M.,
and Mitrakou, A. 1991. Hypoglycemia unawareness.
Endocr. Rev. 12:356-371.
3. White, N.H., et al. 1983. Identification of type 1
diabetic patients at increased risk for hypoglyce- mia during intensive insulin therapy. N. Engl. J. Med. 308:485-491.

4. Heller, S.R., and Cryer, P.E. 1991. Reduced neuroendocrine and symptomatic responses to subsequent hypoglycemia after 1 episode of hypoglycemia in nondiabetic humans. Diabetes. 40:223-226.

5. Davis, M.R., Mellman, M., and Shamoon, H. 1992. Further defects in counterregulatory responses induced by recurrent hypoglycemia in IDDM. Diabetes. 41:1335-1340.

6. Davis, M.R., and Shamoon, H. 1991. Counterregulatory adaptation to recurrent hypoglycemia in normal humans. J. Clin. Endocrinol. Metab. 73:995-1001.

7. Powell, A.M., Sherwin, R.S., and Shulman, G.I. 1993. Impaired hormonal responses to hypogly- 
cemia in spontaneously diabetic and recurrently hypoglycemic rats. Reversibility and stimulus specificity of the deficits. J. Clin. Invest. 92:2667-2674.

8. McCrimmon, R.J., et al. 2005. Activation of KATP channels in the ventromedial hypothalamus amplifies counterregulatory hormone responses to hypoglycemia in normal and recurrently hypoglycemic rats. Diabetes. 54:3169-3174.

9. Flanagan, D.E., et al. 2003. Role of corticotrophinreleasing hormone in the impairment of counterregulatory responses to hypoglycemia. Diabetes. 52:605-613.

10. Borg, W.P., et al. 1994. Ventromedial hypothalamic lesions in rats suppress counterregulatory responses to hypoglycemia. J. Clin. Invest. 93:1677-1682.

11. Borg, W.P., Sherwin, R.S., During, M.J., Borg, M.A., and Shulman, G.I. 1995. Local ventromedial hypothalamus glucopenia triggers counterregulatory hormone release. Diabetes. 44:180-184

12. Borg, M.A., Sherwin, R.S., Borg, W.P., Tamborlane, W.V., and Shulman, G.I. 1997. Local ventromedial hypothalamus glucose perfusion blocks counterregulation during systemic hypoglycemia in awake rats. J. Clin. Invest. 99:361-365.

13. Frizzell, R.T., et al. 1993. Counterregulation during hypoglycemia is directed by widespread brain regions. Diabetes. 42:1253-1261.

14. Oomura, Y., Ono, T., Ooyama, H., and Wayner, M.J. 1969. Glucose and osmosensitive neurones of the rat hypothalamus. Nature. 222:282-284.

15. Sanders, N.M., Dunn-Meynell, A.A., and Levin, B.E. 2004. Third ventricular alloxan reversibly impairs glucose counterregulatory responses. Diabetes. 53:1230-1236.

16. Yang, X.J., Kow, L.M., Funabashi, T., and Mobbs, C.V. 1999. Hypothalamic glucose sensor. Similarities to and differences from pancreatic beta-cell mechanisms. Diabetes. 48:1763-1772.

17. Hevener, A.L., Bergman, R.N., and Donovan, C.M. 1997. Novel glucosensor for hypoglycemic detection localized to the portal vein. Diabetes. 46:1521-1525.

18. Hevener, A.L., Bergman, R.N., and Donovan, C.M 2001. Hypoglycemic detection does not occur in the hepatic artery or liver: findings consistent with a portal vein glucosensor locus. Diabetes. 50:399-403.

19. Ashford, M.L., Boden, P.R., and Treherne, J.M. 1990 Tolbutamide excites rat glucoreceptive ventromedial hypothalamic neurones by indirect inhibition of ATP-K+ channels. Br. J. Pharmacol. 101:531-540.

20. Song, Z., and Routh, V.H. 2005. Differential effects of glucose and lactate on glucosensing neurons in the ventromedial hypothalamic nucleus. Diabetes. 54:15-22.

21. Song, Z., Levin, B.E., McArdle, J.J., Bakhos, N., and Routh, V.H. 2001. Convergence of pre- and postsynaptic influences on glucosensing neurons in the ventromedial hypothalamic nucleus. Diabetes. 50:2673-2681.

22. Evans, M.L., et al. 2004. Hypothalamic ATPsensitive $\mathrm{K}+$ channels play a key role in sensing hypoglycemia and triggering counterregulatory epinephrine and glucagon responses. Diabetes. 53:2542-2551

23. McCrimmon, R.J., et al. 2004. Potential role for AMP-activated protein kinase in hypoglycemia sensing in the ventromedial hypothalamus. Diabetes. 53:1953-1958.

24. Bale, T.L., and Vale, W.W. 2004. CRF and CRF receptors: role in stress responsivity and other behaviors. Ann. Rev. Pharmacol. Toxicol. 44:525-527.

25. Hoare, S.R.J., Sullivan, S.K., Fan, J., Khongsaly, K., and Grigoriadis, D.E. 2005. Peptide ligand binding properties of the corticotropin-releasing factor (CRF) type 2 receptor: pharmacology of endogenously expressed receptors, G-protein-coupling sensitivity and determinants of CRF2 receptor selectivity. Peptides. 26:457-470.

26. Lewis, K., et al. 2001. Identification of urocortin III, an additional member of the corticotrophinreleasing factor (CRF) family with high affinity for the CRF-2 receptor. Proc. Natl Acad. Sci. U. S. A. 98:7570-7575.

27. Chalmers, D.T., Lovenberg, T.W., and De Souza, E.B. 1995. Localization of novel corticotrophinreleasing factor receptor 2 (CRF2) mRNA expression to specific subcortical nuclei in rat brain: comparison with CRF1 receptor mRNA expression. J. Neurosci. 15:6340-6350.

28. Routh, V.H. 2002. Glucose-sensing neurons: are they physiologically relevant? Physiol. Behav. 76:403-413.

29. Routh, V.H. 2003. Glucosensing neurons in the ventromedial hypothalamic nucleus (VMN) and hypoglycemia-associated autonomic failure (HAAF). Diabetes Metab. Res. Rev. 19:348-356.

30. Dunn-Meynell, A.A., Routh, V.H., Kang, L., Gaspers, L., and Levin, B.E. 2002. Glucokinase is the likely mediator of glucosensing in both glucoseexcited and glucose-inhibited central neurons. Diabetes. 51:2056-2065.

31. Ritter, S., Dinh, T.T., and Zhang, Y. 2000. Localization of hindbrain glucoreceptive sites controlling food intake and blood glucose. Brain Res. 856:37-47.

32. Li, C., Vaughan, J., Sawchenko, P.E., and Vale, W.W. 2002. Urocortin III-immunoreactive projections in rat brain: partial overlap with sites of type 2 corticotrophin-releasing factor receptor expression. J. Neurosci. 22:991-1001.

33. Reyes, T.M., et al. 2001. Urocortin II: a member of the corticotrophin-releasing factor (CRF) neuropeptide family that is selectively bound by type 2 CRF receptor. Proc. Natl. Acad. Sci. U. S. A. 98:2843-2848.

34. Bale, T.L., et al. 2000. Mice deficient for corticotrophin releasing hormone receptor-2 display anxietylike behavior and are hypersensitive to stress. Nat. Genet. 24:410-414.

35. Timpl, P., et al. 1998. Impaired stress response and reduced anxiety in mice lacking a functional corticotrophin releasing hormone receptor. Nat. Genet. 19:162-166.
36. Pernar, L., Curtis, A.L., Vale, W.W., Rivier,J.E., and Valentino, R.J. 2004. Selective activation of corticotropin-releasing factor-2 receptors on neurochemically identified neurons in the rat dorsal raphe nucleus reveals dual actions. J. Neurosci. 24:1305-1311.

37. Ter Horst, G.J., and Luiten, P.G.M. 1987. Phaseolus vulgaris leuco-agglutinin tracing of intrahypothalamic connections of the lateral, ventromedial, dorsomedial and paraventricular hypothalamic nuclei in the rat. Brain Res. Bull. 18:191-203.

38. Boyle, P.J., Kempers, S.F., O'Connor, A.M., and Nagy, R.J. 1995. Brain glucose uptake and unawareness of hypoglycemia in patients with insulin-dependent diabetes mellitus. N. Engl. J. Med. 333:1726-1731.

39. Kumagai, A.K., Kang, Y.S., Boado, R.J., and Pardridge, W.M. 1995. Upregulation of bloodbrain barrier GLUT1 glucose transporter protein and mRNA in experimental chronic hypoglycemia. Diabetes. 44:1399-1404.

40. Vannucci, R.C., Nardis, E.E., Vannucci, S.J., and Campbell, P.A. 1981. Cerebral carbohydrate and energy metabolism during hypoglycemia in newborn dogs. Am. J. Physiol. 240:R192-R199.

41. Choi, I.Y., Seaquist, E.R., and Gruetter, R. 2003. Effect of hypoglycemia on brain glycogen metabolism in vivo. J. Neurosci. Res. 72:25-32.

42. Davis, S.N., Shavers, C., Costa, F., and MosquedaGarcia, R. 1996. Role of cortisol in the pathogenesis of deficient counterregulation after antecedent hypoglycemia in normal humans. J. Clin. Invest. 98:680-691.

43. Davis, S.N., Shavers, C., Davis, B., and Costa, F. 1997. Prevention of an increase in plasma cortisol during hypoglycemia preserves subsequent counterregulatory responses. J. Clin. Invest. 100:429-438.

44. Sandoval, D.A., Ping, L., Neill, A.R., Morrey, S., and Davis, S.N. 2003. Cortisol acts through central mechanisms to blunt counterregulatory responses to hypoglycemia in conscious rats. Diabetes. 52:2198-2204.

45. Raju, B., McGregor, V.P., and Cryer, P.E. 2003. Cortisol elevations comparable to those that occur during hypoglycemia do not cause hypoglycemia-associated autonomic failure. Diabetes. 52:2083-2089.

46. Goldberg, P.A., et al. 2006. Antecedent hypercortisolemia is not primarily responsible for generating hypoglycemia-associated autonomic failure. Diabetes. 55:1121-1126.

47. Brown, M.R., et al. 1982. Corticotropin-releasing factor: actions on the sympathetic nervous system and metabolism. Endocrinology. 111:928-931.

48. Cunningham, J.J., Meara, P.A., Lee, R.Y., and Bode, H.H. 1988. Chronic intracerebroventricular CRF infusion attenuates ACTH-corticosterone release. Am. J. Physiol. 255:E213-E217.

49. Wang, R., et al. 2004. The regulation of glucoseexcited neurons in the hypothalamic arcuate nucleus by glucose and feeding-relevant peptides. Diabetes. 53:1959-1965. 